Supporting Information for

\title{
Two Isostructural Flexible Porous Coordination Polymers Showing Contrasting Single-component and Mixture Adsorption Properties for Propylene/Propane
}

Xiao-Yun Tian, Hao-Long Zhou, Xue-Wen Zhang, Chao Wang, Ze-Hao Qiu, Dong-Dong Zhou,* and Jie-Peng Zhang*

MOE Key Laboratory of Bioinorganic and Synthetic Chemistry, School of Chemistry, Sun Yat-Sen University, Guangzhou 510275, China

*Email: zhoudd3@mail.sysu.edu.cn; zhangjp7@mail.sysu.edu.cn 


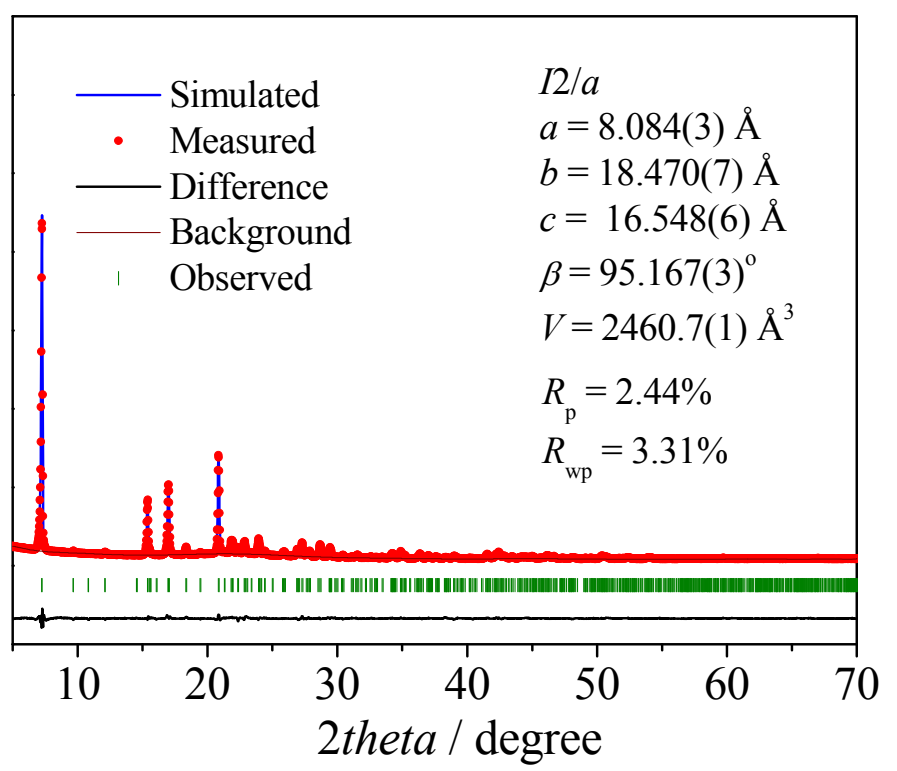

Figure S1. Final Rietveld refinement results of 1'.

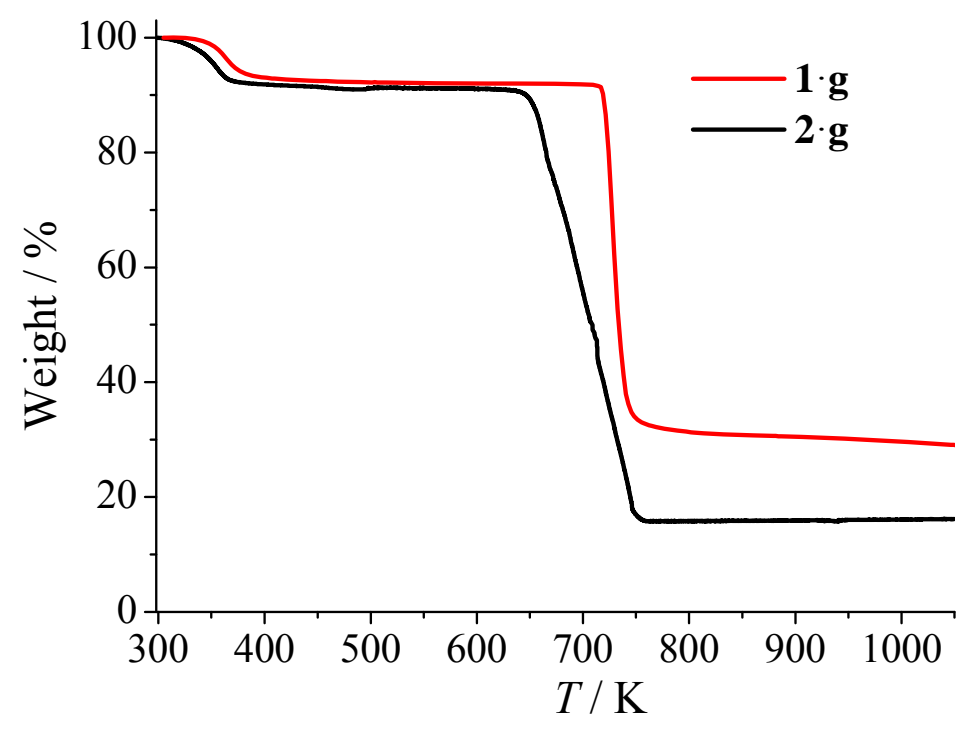

Figure S2. Thermogravimetry curves. 


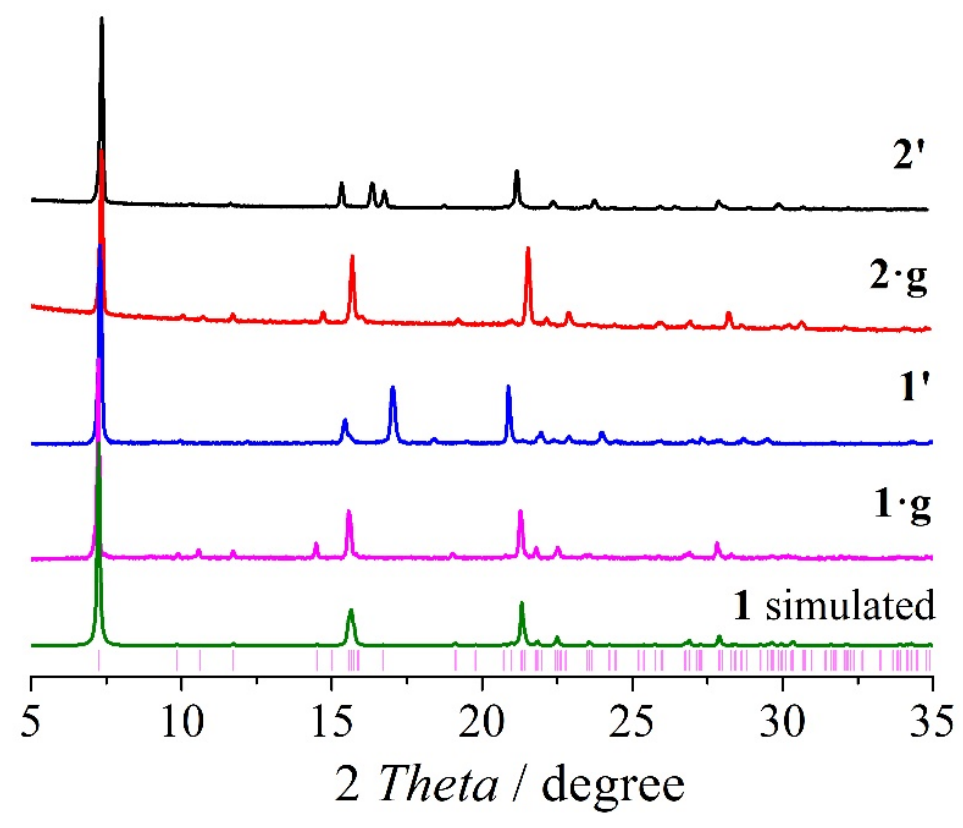

Figure S3. Powder X-ray diffraction patterns.

(a)

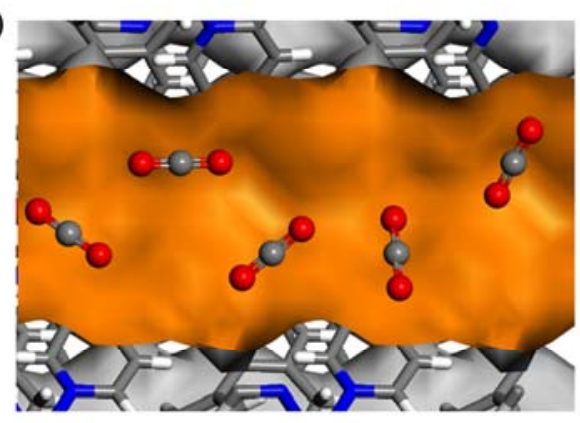

(c)

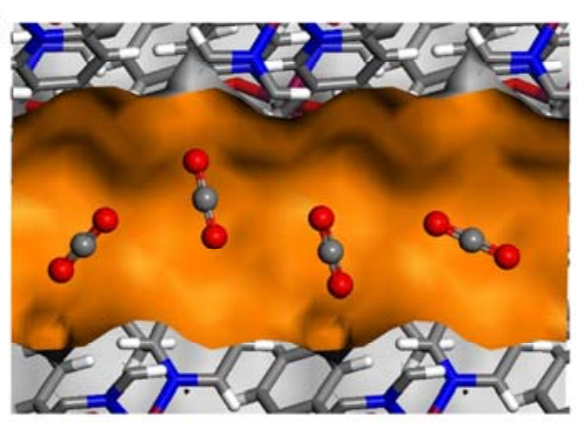

(b)

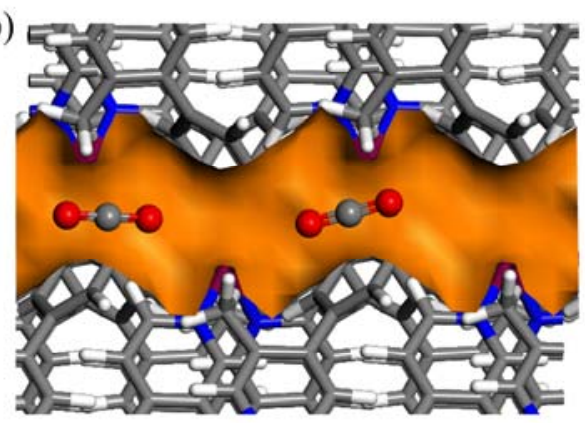

(d)

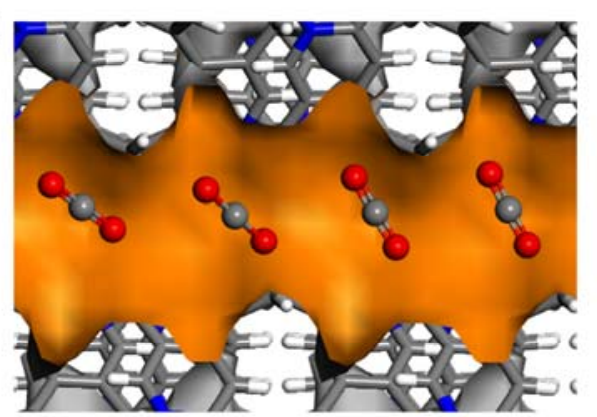

Figure S4. GCMC simulated saturated $\mathrm{CO}_{2}$ adsorption structures for (a) 1, (b) 1', (c) 2 and (d) 2'. 

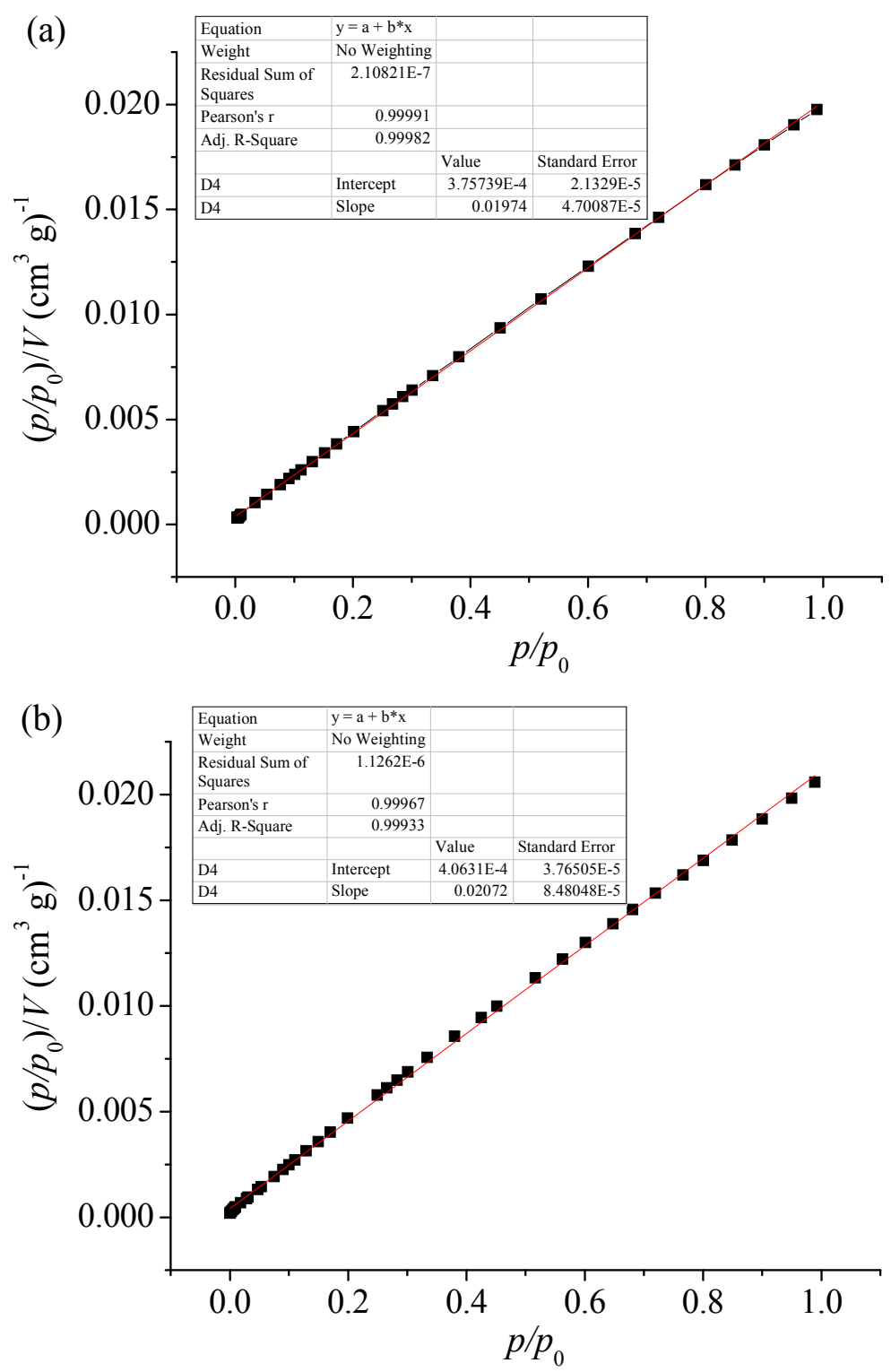

Figure S5. Langmuir fittings of the $\mathrm{CO}_{2}$ isotherms for (a) 1' and (b) 2'. 
(a)

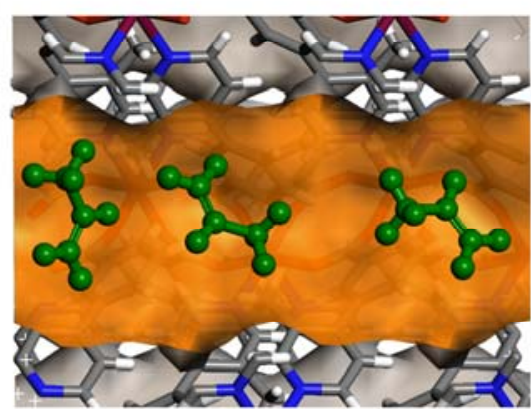

(c)

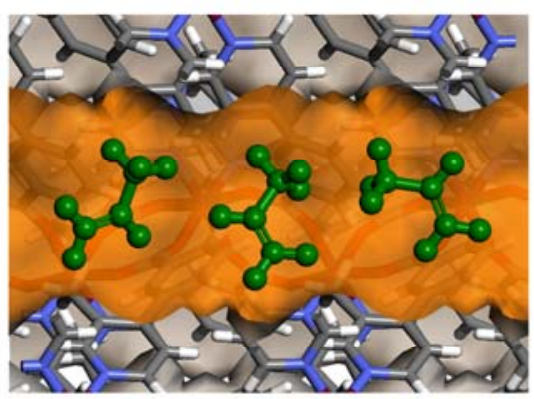

(b)

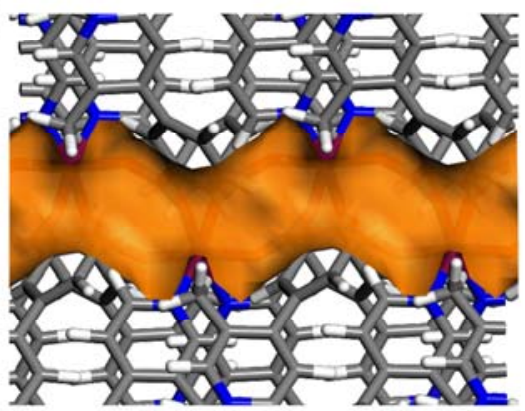

(d)

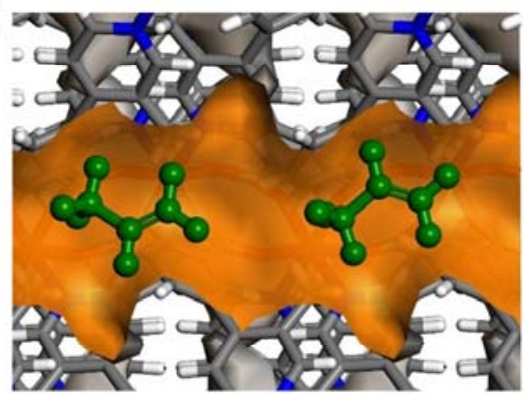

Figure S6. GCMC simulated saturated $\mathrm{C}_{3} \mathrm{H}_{6}$ adsorption structures for (a) 1, (b) 1', (c) 2 and (d) 2'.

(a)

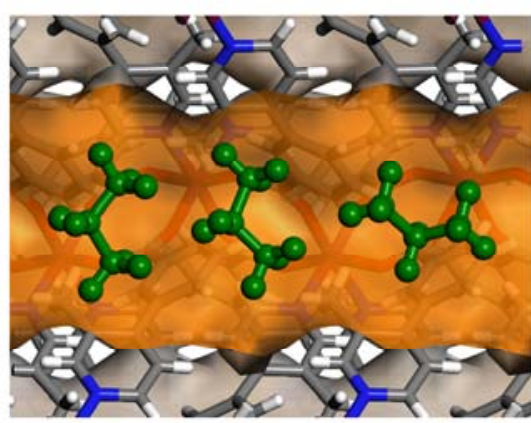

(c)

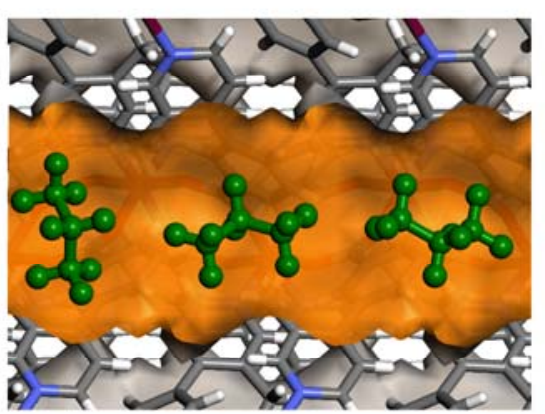

(b)

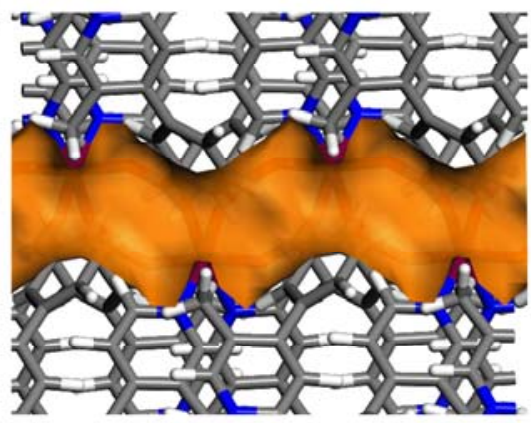

(d)

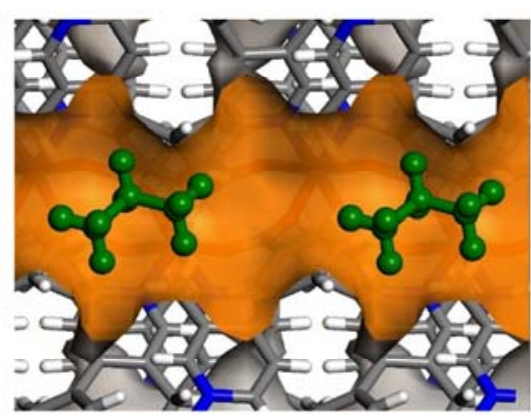

Figure S7. GCMC simulated saturated $\mathrm{C}_{3} \mathrm{H}_{8}$ adsorption structures for (a) 1, (b) 1', (c) 2 and (d) 2'. 



Figure S8. Langmuir fittings of the adsorption isotherms for (a) $\mathrm{C}_{3} \mathrm{H}_{6}$ in $\mathbf{1}^{\prime}$, (b) $\mathrm{C}_{3} \mathrm{H}_{8}$ in $\mathbf{1}^{\prime}$, , (c) $\mathrm{C}_{3} \mathrm{H}_{6}$ in 2', and (d) $\mathrm{C}_{3} \mathrm{H}_{8}$ in 2'. 

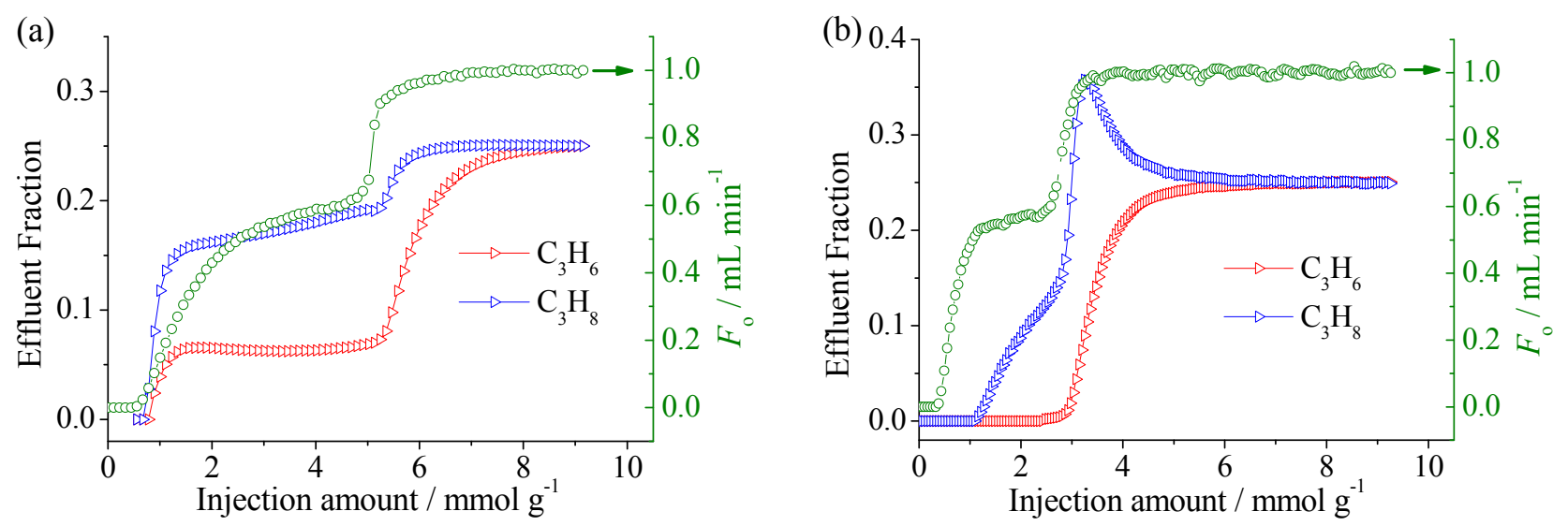

Figure S9. Effluent fractions of $\mathrm{C}_{3} \mathrm{H}_{6} / \mathrm{C}_{3} \mathrm{H}_{8}$ and total flow rates $\left(\mathrm{C}_{3} \mathrm{H}_{6}+\mathrm{C}_{3} \mathrm{H}_{8}+\mathrm{He}\right)$ for (a) 1' and (b) 2'.
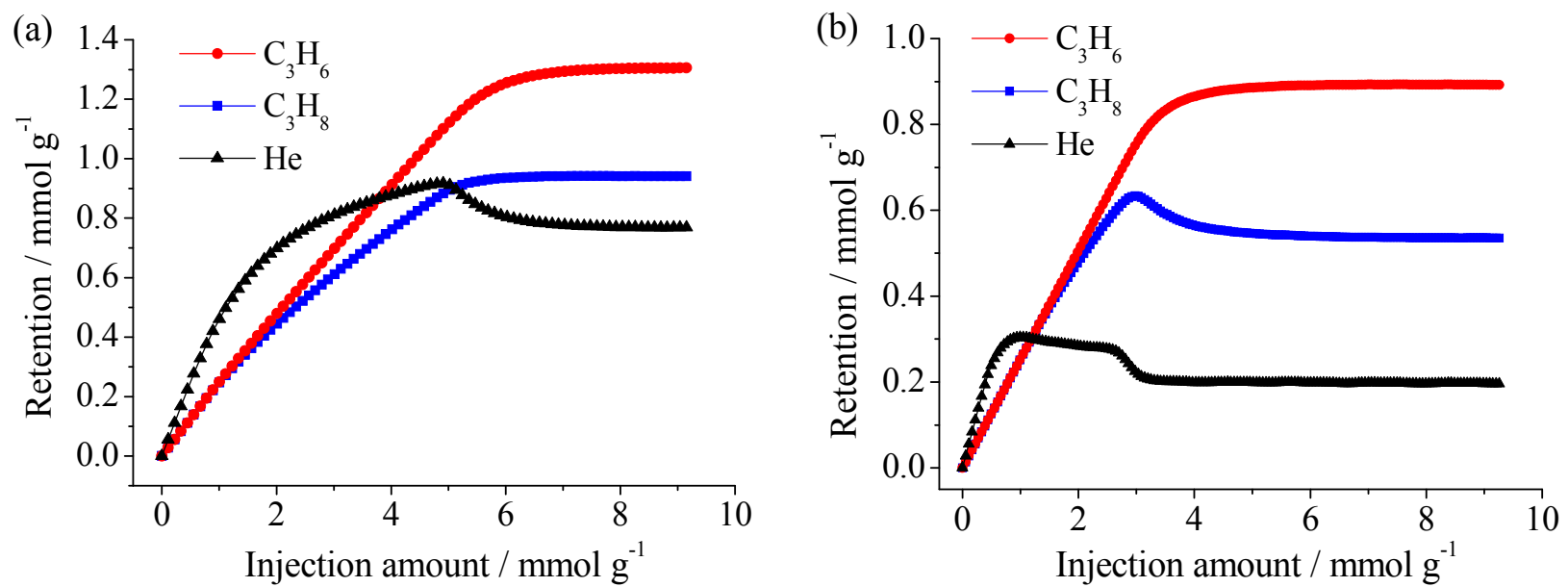

Figure S10. Retention curves for (a) 1' and (b) 2'.
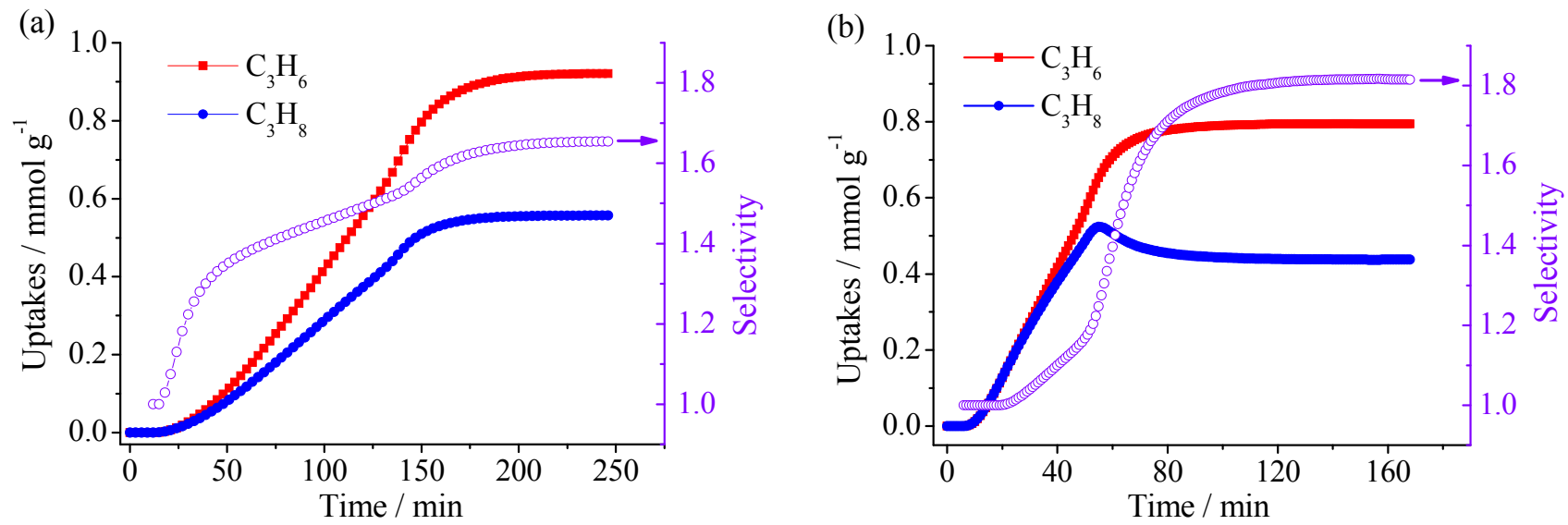

Figure S11. $\mathrm{C}_{3} \mathrm{H}_{6} / \mathrm{C}_{3} \mathrm{H}_{8}$ adsorption amounts and $\mathrm{C}_{3} \mathrm{H}_{6} / \mathrm{C}_{3} \mathrm{H}_{8}$ selectivity for (a) 1' and (b) 2'. 
Table S1. Crystal data and structure refinements results.

\begin{tabular}{|c|c|c|c|c|}
\hline Compound & $1 \cdot g$ & 1' & $2 \cdot g$ & $2 '$ \\
\hline Method & single crystal & powder & single crystal & single crystal \\
\hline Formula & $\mathrm{C}_{26} \mathrm{H}_{20} \mathrm{MnN}_{2} \mathrm{O}_{4}$ & $\mathrm{C}_{26} \mathrm{H}_{20} \mathrm{MnN}_{2} \mathrm{O}_{4}$ & $\mathrm{C}_{26} \mathrm{CoH}_{20} \mathrm{~N}_{2} \mathrm{O}_{4}$ & $\mathrm{C}_{26} \mathrm{CoH}_{20} \mathrm{~N}_{2} \mathrm{O}_{4}$ \\
\hline Formula weight & 479.38 & 479.38 & 483.37 & 483.37 \\
\hline Temperature / K & 170 & 298 & 150 & 150 \\
\hline Space group & $I 2 / a$ & $I 2 / a$ & $I 2 / a$ & $I 2 / a$ \\
\hline$a / \AA$ & $8.4731(2)$ & $8.084(3)$ & $8.5301(1)$ & $8.5281(3)$ \\
\hline$b / \AA$ & $16.6335(3)$ & $18.470(7)$ & $16.3192(3)$ & $16.6993(8)$ \\
\hline$c / \AA$ & $17.9553(3)$ & $16.548(6)$ & $17.6416(3)$ & $16.9482(9)$ \\
\hline$\beta /{ }^{\circ}$ & $92.698(2)$ & 95.167(3) & 92.711(1) & $94.423(4)$ \\
\hline$V / \AA^{3}$ & $2527.78(8)$ & $2460.7(1)$ & $2453.05(7)$ & $2406.5(2)$ \\
\hline$Z$ & 4 & 4 & 4 & 4 \\
\hline$D_{\mathrm{c}} / \mathrm{g} \mathrm{cm}^{-3}$ & 1.260 & 1.294 & 1.309 & 1.334 \\
\hline$R_{\text {int }}$ & 0.0265 & I & 0.0170 & 0.0379 \\
\hline$R_{1}[I>2 \sigma(I)]$ & 0.0315 & $R_{\mathrm{p}}=2.44 \%$ & 0.0356 & 0.0470 \\
\hline$w R_{2}[I>2 \sigma(I)]$ & 0.0881 & 1 & 0.0932 & 0.1219 \\
\hline$R_{1}$ (all data) & 0.0323 & / & 0.0374 & 0.0550 \\
\hline$w R_{2}$ (all data) & 0.0886 & $R_{\mathrm{wp}}=3.31 \%$ & 0.0945 & 0.1268 \\
\hline GOF & 1.100 & 1 & 1.038 & 1.058 \\
\hline
\end{tabular}

$R_{1}=\sum|| F_{\mathrm{o}}|-| F_{\mathrm{c}}|| / \sum\left|F_{\mathrm{o}}\right|$

$w R_{2}=\left\{\sum w\left[\left(F_{\mathrm{o}}\right)^{2}-\left(F_{\mathrm{c}}\right)^{2}\right]^{2} / \sum w\left[\left(F_{\mathrm{o}}\right)^{2}\right]^{2}\right\}^{1 / 2}$

$R_{\mathrm{p}}=\Sigma\left|\mathrm{cY}^{\mathrm{sim}}\left(2 \theta_{\mathrm{i}}\right)-\mathrm{I}^{\mathrm{exp}}\left(2 \theta_{\mathrm{i}}\right)+\mathrm{Y}^{\mathrm{back}}\left(2 \theta_{\mathrm{i}}\right)\right| / \Sigma\left|\mathrm{I}^{\mathrm{exp}}\left(2 \theta_{\mathrm{i}}\right)\right|$

$R_{\mathrm{wp}}=\left\{1 / \mathrm{I}^{\mathrm{exp}}\left(2 \theta_{\mathrm{i}}\right)\left[\mathrm{cY}{ }^{\operatorname{sim}}\left(2 \theta_{\mathrm{i}}\right)-\mathrm{I}^{\mathrm{exp}}\left(2 \theta_{\mathrm{i}}\right)+\mathrm{Y}^{\mathrm{back}}\left(2 \theta_{\mathrm{i}}\right)\right] 2 / \Sigma 1 / \mathrm{I}^{\exp }\left(2 \theta_{\mathrm{i}}\right)\left[\mathrm{I}^{\mathrm{exp}}\left(2 \theta_{\mathrm{i}}\right)\right]^{2}\right\}^{1 / 2}$ 
Table S2. Comparison of the saturated $\mathrm{CO}_{2}$ uptakes obtained by different methods.

\begin{tabular}{|c|c|c|c|c|}
\hline Material & \multicolumn{2}{|c|}{ MCF-56 } & \multicolumn{2}{|c|}{ MCF-57 } \\
\hline State & 1 & $1^{\prime}$ & 2 & $2^{\prime}$ \\
\hline Void ratio $(\%)$ & 18.6 & 5.7 & 17.8 & 12.9 \\
\hline Crystal density $\left(\mathrm{g} \mathrm{cm}^{-3}\right)$ & 1.260 & 1.294 & 1.309 & 1.334 \\
\hline $\begin{array}{l}\text { Saturated uptake from liquid } \\
\text { density }\left(\mathrm{mmol} \mathrm{g}^{-1}\right)\end{array}$ & 3.70 & 1.11 & 3.41 & 2.43 \\
\hline $\begin{array}{l}\text { Saturated uptake from GCMC } \\
\left(\mathrm{mmol} \mathrm{g}^{-1}\right)\end{array}$ & 2.35 & 1.11 & 2.13 & 2.07 \\
\hline $\begin{array}{l}\text { Saturated uptake from GCMC } \\
\left(\mathrm{CO}_{2} \text { per unit cell }\right)\end{array}$ & $36 / 8$ & $17 / 8$ & $33 / 8$ & $32 / 8$ \\
\hline $\begin{array}{l}\text { Experimental uptakes } \\
\left(\mathrm{mmol} \mathrm{g}^{-1}\right)\end{array}$ & \multicolumn{2}{|c|}{2.24} & \multicolumn{2}{|c|}{2.15} \\
\hline
\end{tabular}

Table S3. Comparison of the saturated $\mathrm{C}_{3} \mathrm{H}_{6}$ and $\mathrm{C}_{3} \mathrm{H}_{8}$ uptakes obtained by different methods.

\begin{tabular}{|c|c|c|c|c|}
\hline Material & \multicolumn{2}{|c|}{ MCF-56 } & \multicolumn{2}{|c|}{ MCF-57 } \\
\hline State & 1 & $\mathbf{1}^{\prime}$ & 2 & $2^{\prime}$ \\
\hline $\mathrm{C}_{3} \mathrm{H}_{6}$ from $\mathrm{GCMC}\left(\mathrm{mmol} \mathrm{g}^{-1}\right)$ & 1.56 & 0 & 1.55 & 1.03 \\
\hline $\mathrm{C}_{3} \mathrm{H}_{6} /$ cell from $\mathrm{GCMC}$ & 3 & 0 & 3 & 2 \\
\hline $\begin{array}{l}\text { Experimental } \mathrm{C}_{3} \mathrm{H}_{6} \text { at } 298 \mathrm{~K} \text { and } \\
1 \mathrm{bar} / \text { the saturated uptake fitted } \\
\text { by Langmuir model }\left(\mathrm{mmol} \mathrm{g}^{-1}\right)\end{array}$ & \multicolumn{2}{|c|}{$1.42 / 1.56$} & \multicolumn{2}{|c|}{$1.13 / 1.27$} \\
\hline $\mathrm{C}_{3} \mathrm{H}_{8}$ from $\mathrm{GCMC}\left(\mathrm{mmol} \mathrm{g}^{-1}\right)$ & 1.56 & 0 & 1.55 & 1.03 \\
\hline $\mathrm{C}_{3} \mathrm{H}_{8} /$ cell from GCMC & 3 & 0 & 3 & 2 \\
\hline $\begin{array}{l}\text { Experimental } \mathrm{C}_{3} \mathrm{H}_{8} \text { at } 298 \mathrm{~K} \text { and } \\
1 \mathrm{bar} / \text { the saturated uptake fitted } \\
\text { by Langmuir model }\left(\mathrm{mmol} \mathrm{g}^{-1}\right)\end{array}$ & \multicolumn{2}{|c|}{$1.33 / 1.59$} & \multicolumn{2}{|c|}{$1.10 / 1.32$} \\
\hline
\end{tabular}

